\title{
Magnetocapacitance effect and magnetoelectric coupling in type-II multiferroic $\mathrm{HoFeWO}_{6}$
}

\author{
Moein Adnani $\odot,{ }^{1, *}$ Melissa Gooch $\odot,{ }^{1}$ Liangzi Deng $\odot,{ }^{1}$ Stefano Agrestini $\odot,{ }^{2,3}$ Javier Herrero-Martin $\odot,{ }^{2}$ Hung-Cheng Wu, ${ }^{1,4}$ \\ Chung-Kai Chang, ${ }^{5}$ Taha Salavati-fard, ${ }^{6}$ Narayan Poudel $\odot,{ }^{1, \dagger}$ José Luis García-Muñoz $\odot,{ }^{7}$ Samira Daneshmandi $\odot,{ }^{1}$ \\ Zheng Wu $\odot,{ }^{1}$ Lars C. Grabow $\odot,{ }^{6}$ Yen-Chung Lai, ${ }^{5}$ Hung-Duen Yang $\odot,{ }^{4}$ Eric Pellegrin $\odot,{ }^{2, \ddagger}$ and Ching-Wu Chu $\odot^{1,8,8}$ \\ ${ }^{1}$ Texas Center for Superconductivity and Department of Physics, University of Houston, Houston, Texas 77204, USA \\ ${ }^{2}$ ALBA Synchrotron Light Source, E-08290 Cerdanyola del Vallès, Barcelona, Spain \\ ${ }^{3}$ Diamond Light Source, Harwell Science and Innovation Campus, Didcot, Oxfordshire OX11 ODE, United Kingdom \\ ${ }^{4}$ Department of Physics and Center of Crystal Research, National Sun Yat-Sen University, Kaohsiung, 80424, Taiwan \\ ${ }^{5}$ National Synchrotron Radiation Research Center, Hsinchu, 30076, Taiwan \\ ${ }^{6}$ Texas Center for Superconductivity and William A. Brookshire Department of Chemical and Biomolecular Engineering, \\ University of Houston, Houston, Texas 77204, USA \\ ${ }^{7}$ Institut de Ciència de Materials de Barcelona (ICMAB-CSIC), Campus Universitari de Bellaterra, 08193 Bellaterra, Spain \\ ${ }^{8}$ Lawrence Berkeley National Laboratory, Berkeley, California 94720, USA
}

(Received 27 October 2020; revised 24 December 2020; accepted 26 February 2021; published 17 March 2021)

\begin{abstract}
We have investigated the multiferroicity and magnetoelectric (ME) coupling in $\mathrm{HoFeWO}_{6}$. With a noncentrosymmetric polar structure (space group $P n a 2_{1}$ ) at room temperature, this compound shows an onset of electric polarization with an antiferromagnetic ordering at the Néel temperature $\left(T_{N}\right)$ of $17.8 \mathrm{~K}$. The magnetic properties of the polycrystalline samples were studied by DC and AC magnetization and heat capacity measurements. The metamagnetic behavior at low temperatures was found to be directly related to the dielectric properties of the compound. In particular, field-dependent measurements of capacitance show a magnetocapacitance (MC) effect with double-hysteresis loop behavior in direct correspondence with the magnetization. Our x-ray diffraction results show the $P n a 2_{1}$ structure down to $8 \mathrm{~K}$ and suggest the absence of a structural phase transition across $T_{N}$. Soft $\mathrm{X}$-ray absorption spectroscopy and soft X-ray magnetic circular dichroism (XMCD) measurements at the Fe $L_{2,3}$ and Ho $M_{4,5}$ edges revealed the oxidation state of Fe and Ho cations to be $3+$. Fe $L_{2,3}$ XMCD further shows that $\mathrm{Fe}^{3+}$ cations are antiferromagnetically ordered in a noncollinear fashion with spins arranged $90^{\circ}$ with respect to each other. Our findings show that $\mathrm{HoFeWO}_{6}$ is a type-II multiferroic exhibiting a MC effect. The observed MC effect and the change in polarization by the magnetic field, as well as their direct correspondence with magnetization, further support the strong ME coupling in this compound.
\end{abstract}

DOI: 10.1103/PhysRevB.103.094110

\section{INTRODUCTION}

Materials containing two or more ferroic orders have been the center of attention in the scientific community since they have great potential for devices with multiple controlling parameters as well as exhibiting novel physical phenomena and properties. This class of materials, formerly known as ferroelectromagnets and now as multiferroics, have long been known and extensively studied [1]. Interest in multiferroics was renewed in particular following the experimental observation of spin-induced polarization in rare-earth manganites and the theoretical explanation of its origin [2-9].

An interesting physical property exhibited by these compounds is magnetoelectric (ME) coupling or the mutual

\footnotetext{
*madnanit@uh.edu

${ }^{\dagger}$ Present address: Idaho National Laboratory, Idaho Falls, Idaho 83415, USA.

‡Present address: Carl Zeiss SMT GmbH, SMT-ETRC, 73447 Oberkochen, Germany.

§cwchu@uh.edu
}

coupling between magnetic ordering and polarization [10,11]. One way to indirectly investigate such coupling between spin and charge degree of freedom is through the magnetocapacitance (MC) effect.

Because of the common origin of ferroelectric polarization and magnetic ordering in type-II multiferroics, it is expected that the coupling between magnetic and ferroelectric ordering is relatively strong. Among the type-II multiferroics, orthorhombic rare-earth manganites, which have a nonpolar crystal structure in the paramagnetic region, have been investigated intensively $[7-9,12,13]$ and in fact, most known type-II multiferroics are centrosymmetric in the paramagnetic region.

Among materials having noncentrosymmetric crystal structure and exhibiting multiferroicity, well-known examples are $\mathrm{BiFeO}_{3}$ and hexagonal $\mathrm{YMnO}_{3}$. They are categorized, however, as type-I multiferroics since their ferroelectricity and magnetic ordering have separate origins. While $\mathrm{Fe}^{3+}$ and $\mathrm{Mn}^{3+}$ are responsible for the magnetic state in the respective compounds, the ferroelectricity in $\mathrm{BiFeO}_{3}$ is caused by the lone pair electrons of the $\mathrm{Bi}^{3+}$ ions and that in $\mathrm{YMnO}_{3}$ is of improper geometric nature, in which topology determines its unconventional polarization domain properties [14-18]. 
Several compounds with a noncentrosymmetric polar structure have also been reported to be magnetoelectric multiferroics, e.g., $M_{2} \mathrm{Mo}_{3} \mathrm{O}_{8} \quad(M=\mathrm{Fe}, \mathrm{Mn})$ [19-21], $\mathrm{CaBaCo}_{4} \mathrm{O}_{7}$ [22-24], $\mathrm{Ni}_{3} \mathrm{TeO}_{6}$ [25-27], and recently $R \mathrm{FeWO}_{6}(R=\mathrm{Dy}, \mathrm{Eu}, \mathrm{Tb}$, and $\mathrm{Y})$ [28]. All of these compounds have a broken inversion symmetry in their paramagnetic region driven by the chemical ordering of their cations, and they show a change in polarization at the magnetic ordering temperature without any sign of structural phase transition, in which the polarization is coupled to the magnetic field.

It was proposed that these compounds should be classified as type III, a new class of multiferroics, in order to distinguish them from type II, in which the paramagnetic region is centrosymmetric and the magnetic ordering breaks the inversion symmetry, inducing a spontaneous polarization that is coupled to the magnetic field [29].

In aeschynite-type materials with the general formula of $R M M^{\prime} \mathrm{O}_{6}\left(R=\right.$ rare-earth; $M, M^{\prime}=$ transition metals), due to symmetry constraints, the crystal structure will adopt a polar space group, $P n a 2_{1}$ (No. 33), if $M$ and $M^{\prime}$ are ordered [30]. It was previously reported that the oxides of $R M \mathrm{WO}_{6}(R=$ rareearth element and $M=\mathrm{V}, \mathrm{Cr}$, and $\mathrm{Fe}$ ) have an ordered arrangement of $M^{3+}$ and $\mathrm{W}^{6+}$ and that they show an antiferromagnetic (AFM) ordering at low temperature [31]. In addition, it was recently shown by Ghara et al. [28] that the compounds of $R \mathrm{FeWO}_{6}(R=\mathrm{Dy}, \mathrm{Eu}, \mathrm{Tb}$, and $\mathrm{Y})$ exhibit magnetoelectric multiferroicity. Their neutron diffraction study on DyFeWO revealed a commensurate noncollinear arrangement of $\mathrm{Fe}^{3+}$ and $\mathrm{Dy}^{3+}$ spins with the magnetic space group $\mathrm{C}_{a} \mathrm{c}$.

Here we studied the multiferroic behavior of polycrystalline $\mathrm{HoFeWO}_{6}$ samples. The quality and crystal structure of the samples were confirmed using synchrotron x-ray diffraction (XRD). The x-ray absorption spectroscopy (XAS) measurements confirmed the $3+$ valence state of the $\mathrm{Fe}$ and Ho cations. The X-ray magnetic circular dichroism (XMCD) spectrum on the $\mathrm{Fe} L_{2,3}$ edge showed that the $\mathrm{Fe}^{3+}$ spins are arranged noncollinearly at angles of $90^{\circ}$ with respect to each other. Magnetic, dielectric, and polarization measurements showed that the compound undergoes an AFM ordering at the Néel temperature $\left(T_{N}\right)$ of $17.8 \mathrm{~K}$, accompanied by the onset of a ferroelectric transition at the same temperature. The magnetoelectric coupling was indirectly investigated further by measuring the capacitance as a function of the magnetic field. The direct correspondence of its capacitance and magnetization as functions of the magnetic field and the response of its polarization to the magnetic field indicates the presence of strong ME coupling in this compound.

\section{EXPERIMENT}

Polycrystalline samples of $\mathrm{HoFeWO}_{6}$ were prepared using a conventional solid-state reaction method. To avoid the formation of $\mathrm{Fe}_{3} \mathrm{O}_{4}$ at high temperature, holmium orthoferrite $\left(\mathrm{HoFeO}_{3}\right)$ was synthesized first and then mixed with tungstate $\left(\mathrm{WO}_{3}\right.$ ) [31]. To synthesize $\mathrm{HoFeO}_{3}$, stoichiometric amounts of $\mathrm{Ho}_{2} \mathrm{O}_{3}$ and $\mathrm{Fe}_{2} \mathrm{O}_{3}$ were ground thoroughly and pressed into pellets. Before mixing the precursors, the $\mathrm{Ho}_{2} \mathrm{O}_{3}$ powder was preheated at $900{ }^{\circ} \mathrm{C}$ overnight. The pellets were then treated at $1350{ }^{\circ} \mathrm{C}$ overnight with intermittent cycles of grinding and pressing. The quality of the treated $\mathrm{HoFeO}_{3}$ was checked using XRD and, after confirming its phase purity, it was then mixed with $\mathrm{WO}_{3}$ powder in stoichiometric ratio. The mixture was pelletized and treated at $1050{ }^{\circ} \mathrm{C}$ overnight under flowing argon with intermittent cycles of grinding and pressing. Synthesis in air was attempted for the second step but we could not obtain the desired phase.

Room- and low-temperature synchrotron powder XRD measurements were collected from the as-prepared compound with wavelength $\lambda=0.539$ and $0.82656 \AA$, respectively. The Rietveld refinement analysis of the XRD data was carried out using GSAS-II software [32] and using the orthorhombic space group Pna2 1 (No. 33).

XMCD measurements at the Fe $L_{2,3}$ and Ho $M_{4,5}$ edges were performed at the BL29 BOREAS beamline of the ALBA Synchrotron Radiation Facility in Barcelona [33].

The unit cell provided by XRD measurement at $300 \mathrm{~K}$ was used for the density functional theory (DFT) calculations. The stoichiometric unit cell contains 36 atoms: $4 \mathrm{Ho}$, $4 \mathrm{~W}, 4 \mathrm{Fe}$, and $24 \mathrm{O}$ atoms. All periodic, spin-polarized DFT calculations were carried out using the Vienna $a b$ initio simulation package (VASP) [34-36] version 5.4.1 and the atomic simulation environment (ASE) [37]. Exchange and correlation effects were taken into account using the PBE density functional introduced by Perdew, Burke, and Ernzerhof [38]. The wave function of the valance electrons was expanded into a plane wave basis set with an energy cutoff of $400 \mathrm{eV}$, while the core electrons were represented using the projector augmented wave function (PAW) method $[39,40]$. Given the size of the simulation box, a $\Gamma$-centered $(2,4,3) k$ point grid was considered for sampling the reciprocal space. A Gaussian-smearing scheme with $k_{B} T=0.2 \mathrm{eV}$ was used to treat discontinuities at the Fermi level, and the total energy was extrapolated to $k_{B} T=0.0 \mathrm{eV}$. Self-consistent field calculations were performed until a determination of energy accuracy to $10^{-5} \mathrm{eV}$ was achieved. Geometry optimizations were performed with the conjugate-gradient algorithm until the maximum force acting on atoms was less than $0.02 \mathrm{eV} / \AA$. To include exact exchange effects, which are known to be able to widen the band gap and provide a better density of states and band structure, the HSE06 hybrid density functional [41] was employed for single-point wave function calculations. The electronic properties of the system were obtained from the HSE06 wave function. We recognize that spin-polarized collinear DFT calculations neglect the magnetic ordering to keep the computations tractable and may therefore not be able to predict a magnetically induced structural phase transition, but they can predict electronic properties and the stability or metastability of the structure with reasonable accuracy.

DC and AC magnetic measurements were performed using the Quantum Design Magnetic Property Measurement System (MPMS3). For dielectric constant and polarization measurements, we prepared a thin $(\sim 1 \mathrm{~mm})$ disk-shaped sample, covered both faces with silver paint to serve as electrodes, and attached thin Pt wires to each face. The capacitance of the sample was measured using a precision capacitance bridge $(\mathrm{AH} 2500 \mathrm{~A})$ at the frequency of $1 \mathrm{kHz}$ during heating at the rate of $1 \mathrm{~K} / \mathrm{min}$. The electric polarization was measured via the pyrocurrent $\left(I_{\text {pyro }}\right)$ method using a Keithley K6517A electrometer. To measure the $I_{\mathrm{pyro}}(T)$, the sample was cooled 

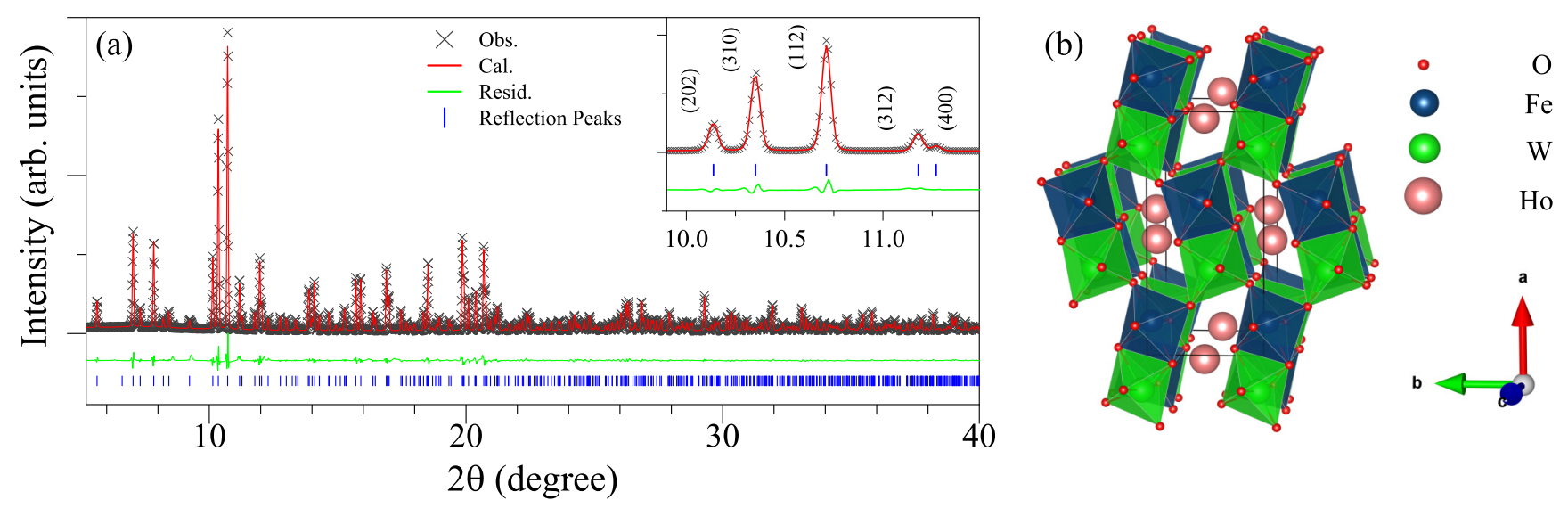

FIG. 1. (a) Rietveld refinement of the room-temperature synchrotron XRD data for $\mathrm{HoFeWO}_{6}$. Crosses: measurement data; red solid line: calculated profile; green solid line: the difference between the observation and the calculated profile; and vertical bars: positions of Bragg peaks. Inset: magnified view of the main peaks. (b) Crystal structure of $\mathrm{HoFeWO}_{6}$.

down under a poling field of $E_{\text {pole }} \sim 270 \mathrm{kV} / \mathrm{m}$. The poling field $E_{\text {pole }}$ lines up the electric dipoles that are formed below the ferroelectric transition. At the lowest temperature of $2 \mathrm{~K}$, the poling field was removed and the desired magnetic field was applied. The $I_{\text {pyro }}$ was measured during subsequent warming at the rate of $2 \mathrm{~K} / \mathrm{min}$. In both the dielectric and polarization measurements, the temperature and the magnetic field were controlled by a Physical Property Measurement System (PPMS, Quantum Design). For magnetocapacitance measurements, we swept the magnetic field inside the PPMS at a rate of $50 \mathrm{Oe} / \mathrm{s}$ and at a constant temperature while continuously measuring the capacitance using the capacitance bridge.

\section{RESULTS AND DISCUSSION}

XRD was performed at room temperature on powder samples of $\mathrm{HoFeWO}_{6}$ and the pattern, along with its Rietveld refinement, is shown in Fig. 1(a). A few additional tiny peaks that could not be indexed or identified and are likely due to impurities were also observed. Considering the high resolution of the synchrotron XRD, we expect the amount of impurities to be less than $2 \%$. The inset to Fig. 1(a) shows a magnified view of the main peaks. The results of the crystal structure parameters are compared with one another in Table I. The crystal structure of the compound, as depicted in Fig. 1(b), consists of edge-sharing dimers of $\mathrm{FeO}_{6}$ and $\mathrm{WO}_{6}$ octahedra that connect to each other through corners to form a three-dimensional framework. The $\mathrm{Ho}^{3+}$ cations occupy the channels in between the octahedra along the $c$ axis. Illustrations of the structure in other directions are presented in Fig. S1 [42].

Low-temperature XRD patterns were also obtained from 30 to $8 \mathrm{~K}$ across the AFM transition. The Rietveld refinement performed on the patterns (not shown) did not have a good fit; however, the profiles were matched with the Bragg reflections, which suggests the absence of a structural phase transition in this temperature region. The structural relaxation calculation by the PBE density functional also shows the absence of a structural transition for this system. We caution that these calculations do not include magnetic ordering of the system and we cannot rule out the possibility of a different observation by noncollinear DFT calculations. However, the consistency among the lattice parameters obtained from both low- and room-temperature XRD and from our DFT calculations (Table II) suggest that $P n a 2_{1}$ is the stable structure and the ground state of this compound, although the possibility of lattice distortion cannot be excluded.

The oxidation state of the $\mathrm{Fe}$ cations in $\mathrm{HoFeWO}_{6}$ was investigated by XAS and XMCD and by spin-polarized DFT calculations, and the results are presented in Fig. 2.

TABLE I. Structural and lattice parameters obtained from the Rietveld refinement of XRD data at $T=300 \mathrm{~K}$.

\begin{tabular}{|c|c|c|c|c|c|c|}
\hline Atom & $x$ & $y$ & $z$ & Occupancy & $U_{\text {iso }}\left(\AA^{2}\right)$ & site \\
\hline Ho & $0.0430(1)$ & $0.4564(3)$ & $0.2476(5)$ & 1 & $0.0179(3)$ & $4 a$ \\
\hline $\mathrm{Fe}$ & $0.1393(5)$ & $0.9633(9)$ & $0.9907(14)$ & 1 & $0.015(1)$ & $4 a$ \\
\hline W & $0.3544(2)$ & $0.4517(3)$ & $0.00350(15)$ & 1 & $0.0181(3)$ & $4 a$ \\
\hline $\mathrm{O} 1$ & $0.966(2)$ & $0.780(4)$ & $0.047(4)$ & 1 & $0.018(2)$ & $4 \mathrm{a}$ \\
\hline $\mathrm{O} 2$ & $0.521(2)$ & $0.250(4)$ & $0.966(4)$ & 1 & $0.018(2)$ & $4 a$ \\
\hline $\mathrm{O} 3$ & $0.217(2)$ & $0.611(4)$ & $0.061(3)$ & 1 & $0.018(2)$ & $4 a$ \\
\hline $\mathrm{O} 4$ & $0.297(2)$ & $0.135(5)$ & $0.933(3)$ & 1 & $0.018(2)$ & $4 a$ \\
\hline O5 & $0.145(2)$ & $0.060(4)$ & $0.263(3)$ & 1 & $0.018(2)$ & $4 a$ \\
\hline O6 & $0.118(2)$ & $0.836(4)$ & $0.742(5)$ & 1 & $0.018(2)$ & $4 a$ \\
\hline \multicolumn{7}{|c|}{$\begin{array}{l}\text { Space group }=P n a 2_{1} ; \text { Lattice parameters: } a=10.973(1) \AA, b=5.1696(5) \AA, c=7.3342(6) \AA, \alpha=\beta=\gamma=90^{\circ} ; \\
\text { Volume }=416.046(5) \AA^{3} ; R_{F}=3.24 \%, R_{F}^{2}=4.64, w R=8.61 \%, R=6.35 \%, \chi^{2}=2.12\end{array}$} \\
\hline
\end{tabular}


TABLE II. Lattice parameters obtained from XRD at $T=300$ and $8 \mathrm{~K}$ and from DFT calculations.

\begin{tabular}{lcccc}
\hline \hline & $a(\AA)$ & $b(\AA)$ & $c(\AA)$ & Vol. $\left(\AA^{3}\right)$ \\
\hline XRD (300 K) & $10.973(1)$ & $5.1696(5)$ & $7.3342(6)$ & $416.05(5)$ \\
XRD (8 K) & $10.9537(4)$ & $5.1574(2)$ & $7.31892(23)$ & $413.463(2)$ \\
DFT & 10.99 & 5.15 & 7.31 & 413.88 \\
\hline \hline
\end{tabular}

The x-ray absorption spectra were collected using circularly polarized light with the photon spin aligned parallel $\left(\sigma^{+}\right)$ and antiparallel $\left(\sigma^{-}\right)$to the magnetic field. The difference spectrum $\left(\sigma^{+}-\sigma^{-}\right)$is called XMCD and the sum spectrum $\left(\sigma^{+}+\sigma^{-}\right)$is called XAS. Results of the Fe $L_{2,3}$ XAS and XMCD measurements on $\mathrm{HoFeWO}_{6}$ performed under a magnetic field of $6 \mathrm{~T}$ at the temperature of $2 \mathrm{~K}$ are shown as blue and red dotted curves, respectively, in Fig. 2(b). The XAS spectrum of $\alpha-\mathrm{Fe}_{2} \mathrm{O}_{3}$ is also shown as a $\mathrm{Fe}^{3+}$ high spin $S=$ $5 / 2$ reference sample [Fig. 2(a)]. We first verified the valence state of the $\mathrm{Fe}$ ions in $\mathrm{HoFeWO}_{6}$. The energy position of the XAS white lines is known to increase systematically with the valence state of the investigated ion [43-46]. The similarity of the energy position of the $\mathrm{Fe} L_{2,3}$ XAS white lines of our sample and that of $\alpha-\mathrm{Fe}_{2} \mathrm{O}_{3}$ demonstrates the trivalent state
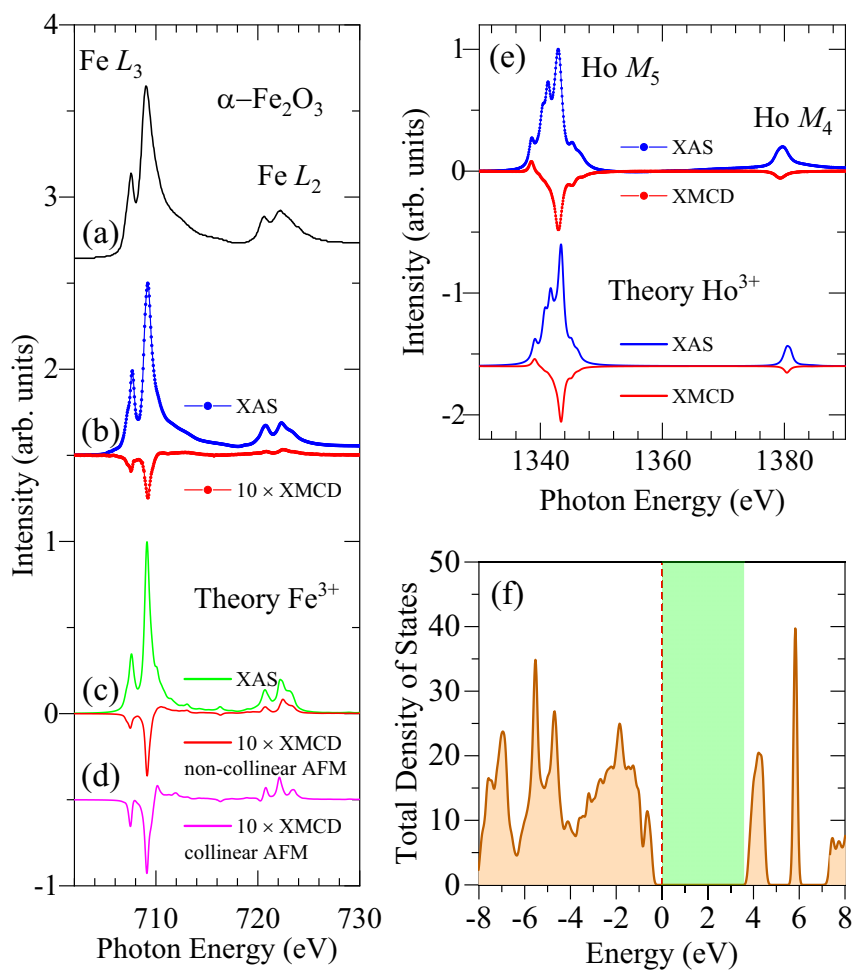

FIG. 2. (a) $\mathrm{Fe} L_{2,3}$ XAS spectrum of $\alpha-\mathrm{Fe}_{2} \mathrm{O}_{3}$, (b) $\mathrm{Fe} L_{2,3}$ XAS (blue) and $\mathrm{XMCD}$ (red) spectra of $\mathrm{HoFeWO}_{6}$ measured at $2 \mathrm{~K}$ under a magnetic field of $6 \mathrm{~T}$, (c) calculated XAS and XMCD spectra for noncollinear AFM order, and (d) calculated XMCD spectrum for collinear AFM order. The XMCD spectra are scaled by a factor of 10 for a clearer view. (e) Ho $M_{4,5}$ XAS (blue dots) and XMCD (red dots) spectra of $\mathrm{HoFeWO}_{6}$ measured at $2 \mathrm{~K}$ under a magnetic field of $6 \mathrm{~T}$, together with the calculated XAS (solid blue line) and XMCD (solid red line) of $\mathrm{Ho}^{3+}$. (f) Total density of states for $\mathrm{HoFeWO}_{6}$. Dashed line: Fermi energy. Highlighted region: insulating gap of $\sim 3.7 \mathrm{eV}$. of the Fe ions in $\mathrm{HoFeWO}_{6}$. Both the XAS and XMCD spectra exhibit a complex line shape called the multiplet structure. This multiplet structure depends on the multiplet interaction, the crystal field, and the hybridization, and as such is highly sensitive to the symmetry of the electronic and magnetic ground states. The multiplet features of XAS are very similar to those of $\alpha-\mathrm{Fe}_{2} \mathrm{O}_{3}$, which demonstrates that the $\mathrm{Fe}^{3+}$ ions in $\mathrm{HoFeWO}_{6}$ have a high spin $S=5 / 2$ state.

Despite the large spin moment, the measured $\mathrm{Fe} L_{2,3}$ $\mathrm{XMCD}$ signal is only $2.5 \%-5 \%$ as intense as XAS, and in Fig. 2(b), a scale factor of 10 was applied for a clearer view of this spectrum. We have used the sum rules for XMCD developed by Thole et al. [47] and Carra et al. [48] to extract from our XMCD data a quantitative estimate of the spin and orbital moments:

$$
\begin{aligned}
m_{l}= & \frac{4}{3} \frac{\int_{L_{2,3}}\left(\sigma^{+}-\sigma^{-}\right) d E}{\int_{L_{2,3}}\left(\sigma^{+}+\sigma^{-}\right) d E} N_{h}, \\
m_{s}+7 T_{z}= & 2 \frac{\int_{L_{3}}\left(\sigma^{+}-\sigma^{-}\right) d E-2 \int_{L_{2}}\left(\sigma^{+}-\sigma^{-}\right) d E}{\int_{L_{2,3}}\left(\sigma^{+}+\sigma^{-}\right) d E} \\
& \times N_{h} \frac{1}{R},
\end{aligned}
$$

where $N_{h}=5$ is the number of holes in the $d$ band and the intra-atomic magnetic dipole moment $T_{z}$ can be assumed to be close to zero because the $d$ shell in $\mathrm{Fe}^{3+}$ is half-filled $\left(3 d^{5}\right)$. The factor $R=0.7$ takes into account the $30 \%$ underestimation of the spin moment in $\mathrm{Fe}^{3+}$ due to the partial mixing of the $L_{3}$ and $L_{2}$ edges induced by the electron-core-hole interaction [49]. Table S1 [42] summarizes the moments obtained by the application of the sum rules. The net magnetic moment of the $\mathrm{Fe}^{3+}$ ion $\left(m_{s}+m_{l}\right)$ at the temperature of $2 \mathrm{~K}$ and under an applied field of $6 \mathrm{~T}$ is about $0.27 \mu_{B} /$ ion. This value is much smaller than the saturated moment of $5 \mu_{B}$, as well as the paramagnetic moment of $4.4 \mu_{B}$ in $\mathrm{Fe}^{3+}$ estimated for $T=2 \mathrm{~K}$ and $B=6 \mathrm{~T}$ considering a Boltzmann population of the many-body energy levels. Such a small moment rules out either a ferromagnetic or a paramagnetic ground states and suggests an AFM ordering of the $\mathrm{Fe}^{3+}$ moments.

To extract more detailed information about the ground state, we performed the well-established configurationinteraction cluster calculations using the XTLS code [50]. The method uses a $\mathrm{FeO}_{6}$ cluster, which explicitly includes the full atomic multiplet interaction, the hybridization of $\mathrm{Fe}$ with the oxygen ligands, and the crystal field acting on the Fe ions. The hybridization strengths and the crystal field parameters were estimated using a charge point model and Harrison's prescription, respectively [51]. The parameter values are reported in [52]. The calculated XAS [Fig. 2(c), green solid curve] for $\mathrm{Fe}^{3+}$ in octahedral coordination is in very good agreement with the experimental XAS result. The calculated XMCD of paramagnetic $\mathrm{Fe}^{3+}$ at $T=2 \mathrm{~K}$ and $B=6 \mathrm{~T}$ is about 15 times larger than the measured XMCD. The small size of the experimental XMCD signal can be explained by an AFM ordering of the $\mathrm{Fe}$ moments in $\mathrm{HoFeWO}_{6}$. In this scenario, the XMCD signal is generated by the net moment induced by the applied magnetic field. To take into account the AFM order, we introduced into the Hamiltonian an exchange field and obtained the XMCD as the average of the spectra 
calculated for opposite directions of the exchange field. An exchange field of about $4 \mathrm{meV}$ is needed to reproduce the size of the experimental XMCD spectrum. We first considered an isotropic collinear AFM order. The angle between the exchange field and the applied magnetic field was varied from $0^{\circ}$ to $90^{\circ}$, but none of the calculations could reproduce the experimental XMCD. As a second step, we considered a collinear AFM order, in which magnetic anisotropy forces the spin to be oriented along a particular axis. Since we are studying a polycrystalline sample, we simulated the experimental data by summing two calculated spectra: one with the exchange field parallel to the magnetic field and one with the exchange field normal to the magnetic field, with a weighting ratio of $1: 2$. This calculated XMCD [Fig. 2(d), magenta solid curve] still does not reproduce the experimental XMCD result at the $L_{2}$ edge. In order to improve the simulation of the XMCD, a canting of the $\mathrm{Fe}$ moments of roughly $45^{\circ}$ must be introduced. In particular, the experimental data can be closely simulated [Fig. 2(c), red solid curve] by considering a noncollinear AFM model, observed by Ghara et al. [28] in $\mathrm{DyFeWO}_{6}$ using neutron diffraction. In this model there are two $\mathrm{Fe}$ sites, $\mathrm{Fe} 1$ and $\mathrm{Fe} 2$, for which spins are arranged antiferromagnetically on two perpendicular planes, $a c$ and $b c$, respectively. The spin of the $\mathrm{Fe} 2$ forms an angle of approximately $45^{\circ}$ with respect to the plane of the Fel site. By considering the noncollinear AFM model, not only is the improvement in the simulation of the XMCD evident at the $L_{2}$ edge, but the intensity ratio for the two features at the XMCD $L_{3}$ edge is also much closer to that in the experimental curve. It should be noted that XMCD cannot probe directly the long-range arrangement of the spins but can only determine the effect of the spin arrangement at a local level. Hence, our XMCD results cannot exclude other strongly noncollinear magnetic orders like helicals or spirals. The calculations show that the $\mathrm{Fe}^{3+}$ ions are in a high spin $S=5 / 2$ state and that the magnetic moment of the single $\mathrm{Fe}^{3+}$ ion is $4.6 \mu_{B}$. The calculations also estimate a net moment of about $0.26 \mu_{B}$ for the $\mathrm{Fe}^{3+}$ ions arranged in the noncollinear AFM order in the presence of an applied field of $6 \mathrm{~T}$, which is in very good agreement with the results of the sum rules.

Figure 2(e) shows the Ho $M_{4,5}$ XAS and XMCD measurements of $\mathrm{HoFeWO}_{6}$ performed under a magnetic field of $6 \mathrm{~T}$ at a temperature of $2 \mathrm{~K}$. To estimate the spin and orbital moments of the rare-earth ions, we applied the sum rules $[47,48]$ for $M_{4,5}$ edges to our XMCD data:

$$
\begin{aligned}
m_{l} & =2 \frac{\int_{M_{4,5}}\left(\sigma^{+}-\sigma^{-}\right) d E}{\int_{M_{4,5}}\left(\sigma^{+}+\sigma^{-}\right) d E} N_{h}^{f}, \\
m_{s}+6 T_{z} & =\frac{2 \int_{M_{5}}\left(\sigma^{+}-\sigma^{-}\right) d E-3 \int_{M_{4}}\left(\sigma^{+}-\sigma^{-}\right) d E}{\int_{M_{4,5}}\left(\sigma^{+}+\sigma^{-}\right) d E} N_{h}^{f} \frac{1}{R},
\end{aligned}
$$

where $N_{h}^{f}$ is the number of holes in the $f$ band and the $R$ factor is 0.96 [53]. We used cluster calculations to determine the intra-atomic dipole moment, and the ratio $T_{z} / S_{z}$ was estimated to be 0.089 . The moments obtained by the application of the sum rules are shown in Table S1 [42].

Both the experimental and the calculated $M_{4,5}$ spectra are displayed in Fig. 2(e). The good agreement between the simulation and the experimental spectra demonstrates that the
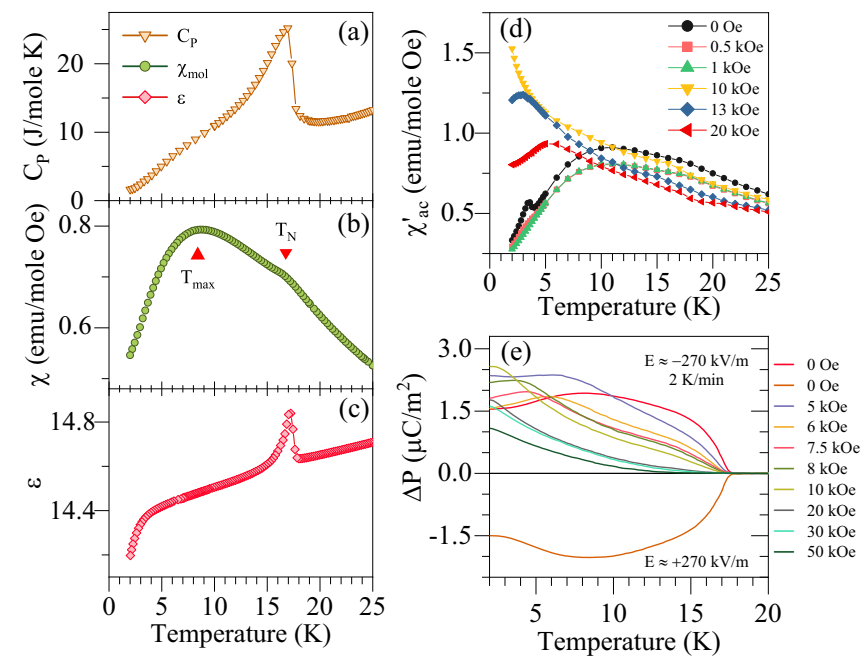

FIG. 3. (a) Heat capacity, (b) (molar) magnetic susceptibility, and (c) dielectric constant as functions of temperature. Heat capacity and dielectric constant were measured during warming without the presence of a magnetic field and magnetic susceptibility was measured under the field-cooled condition and in the presence of $H=1 \mathrm{~T}$. (d) Real part of AC magnetic susceptibility, $\chi_{\mathrm{AC}}(T)$, measured under the frequency of $f=1 \mathrm{kHz}$ and a time-varying magnetic field of $H_{\mathrm{AC}}=2$ Oe and under different external magnetic fields. (e) Electric polarization as a function of temperature, $\Delta P(T)$, under different magnetic fields. To measure the $\Delta P(T)$, the sample was cooled down from $20 \mathrm{~K}$ to the base temperature of $2 \mathrm{~K}$ in the presence of the poling field $\mathrm{E}$. The $\Delta P(T)$ was then measured after removing $\mathrm{E}$ and applying the magnetic field while warming the sample at the rate of $2 \mathrm{~K} / \mathrm{min}$.

valence state of the Ho ions is $3+$. The relevant features of the XAS and XMCD spectra could be captured in our simulation only when an exchange field of at least $2 \mathrm{meV}$ is introduced into the Hamiltonian. In contrast to the Fe $L_{2,3} \mathrm{XMCD}$, the Ho $M_{4,5}$ XMCD cannot distinguish between collinear and noncollinear AFM order.

The spin-polarized DFT calculations were used to estimate the oxidation states by approximating the magnetic moment of each atom without including the effects of spin-orbit coupling, and the results are presented in Table S2 [42]. By comparing these results and the electronic configuration of elemental Ho, $\mathrm{Fe}$, and $\mathrm{W}$ and using Hund's law, one would estimate the oxidation states as $3+, 3+$, and $6+$, respectively. This is quite consistent with our XAS and XMCD results and also in good agreement with previous reports $[28,30]$. The density of states calculations also confirm that orthorhombic $\mathrm{HoFeWO}_{6}$ is an insulator with a wide band gap of $3.7 \mathrm{eV}$, as shown in Fig. 2(f).

The lambda-shaped peak in the heat capacity, shown in Fig. 3(a), and the kink in the DC (molar) magnetic susceptibility $\chi(T)=M / H$, shown in Fig. 3(b), both indicate a second-order phase transition, which can be interpreted as the onset of the long-range AFM ordering of $S=5 / 2 \mathrm{Fe}^{3+}$ cations with a half-filled $d$ shell, as suggested by our XMCD results. By lowering the temperature, susceptibility exhibits a broad maximum at $T_{\max }=10.8 \mathrm{~K}$, below which it continues to decrease to the lowest temperature. This broad maximum is reminiscent of a low-dimensional magnetic system [54]. This 


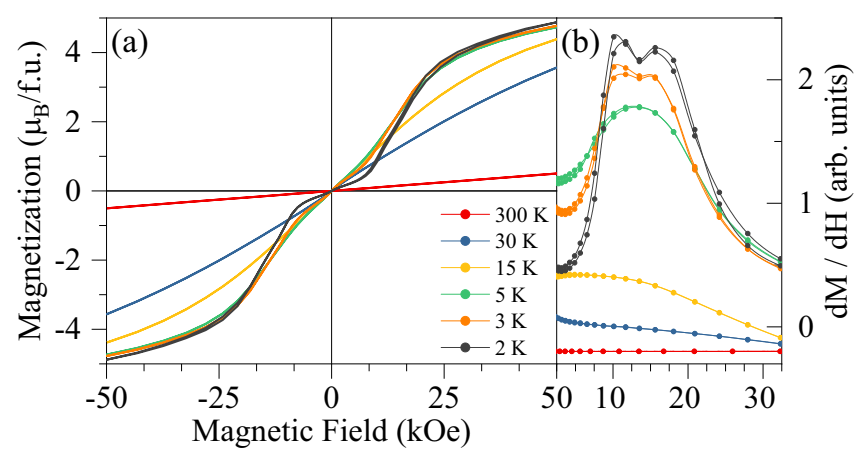

FIG. 4. (a) Field-dependent DC magnetization $M(H)$ at different temperatures and (b) its derivative. For clarity, the derivatives are shifted in the vertical direction.

behavior could be related to the arrangement of $\mathrm{Ho}^{3+}$ ions in the crystal structure since they occupy the channels between the $\mathrm{FeO}_{6}$ and $\mathrm{WO}_{6}$ octahedra along the $c$ direction, creating a quasi-one-dimensional chain. Views of the crystal structure in different orientations can be found in Fig. S1 [42].

As shown in Fig. 3(c), the AFM transition at $17.8 \mathrm{~K}$ is accompanied by a peak in the dielectric constant, measured at the frequency of $f=1 \mathrm{kHz}$, which is consistent with a ferroelectric transition. It has been observed for other compounds of this family that the onset temperature of this peak does not change at different frequencies [28], indicating that the observed anomaly is not caused by relaxation phenomena. The ferroelectric transition is further confirmed by the observation of a switchable polarization, as shown in Fig. 3(e), and of an anomaly in the dielectric loss, as shown below in the inset to Fig. 5(a). These observations favor the proposition that the observed ferroelectricity originates from spin ordering. The observation of ferroelectric polarization in $\mathrm{YFeWO}_{6}$ with a nonmagnetic rare-earth cation indicates that the appearance of ferroelectric polarization in these compounds is due to ordering of $\mathrm{Fe}^{3+}$ [28]. The fact that the $\mathrm{Fe}^{3+}$ spins are arranged noncollinearly indicates that the inverse Dzyaloshinskii-Moriya (DM) interaction is likely the underlying mechanism for breaking the inversion symmetry by magnetic ordering.

The inverse magnetic susceptibility $\chi^{-1}(T)$, shown in Fig. S2 [42], follows the Curie-Weiss (CW) law $\chi^{-1}(T)=3 k_{B}\left(T-\theta_{\mathrm{CW}}\right) / N_{A} \mu_{\mathrm{eff}}^{2}$ in the paramagnetic region. Based on the $\mathrm{CW}$ fit, the effective total magnetic moment was estimated as $\mu_{\text {eff }}=12.58 \mu_{B} /$ f.u., which is in good agreement with the theoretical value of $\mu_{\text {th }}=12.14 \mu_{B} /$ f.u. for the $\mathrm{Fe}^{3+}$ and $\mathrm{Ho}^{3+}$ moments and the $\mathrm{CW}$ temperature of $\theta_{\mathrm{CW}}=-18.88 \mathrm{~K}$. The negative sign also confirms that the dominant interaction below $T_{\mathrm{N}}$ is antiferromagnetic.

Figure 3(d) shows the real part of the AC susceptibility $\chi_{\mathrm{AC}}(T)$ for this compound under different external magnetic fields. A small time-varying magnetic field of $H_{\mathrm{AC}}=2 \mathrm{Oe}$ with the frequency $f=1 \mathrm{kHz}$ is used for measuring the $\chi_{\mathrm{AC}}(T)$. The curve at $0 \mathrm{Oe}$ is, in essence, similar to the DC susceptibility. The broad peak below the $T_{N}$, which, as mentioned previously, is characteristic of low-dimensional magnets, is likely due to the formation of a regime of shortrange correlation between $\mathrm{Ho}^{3+}$ spins, which at further lower (a)

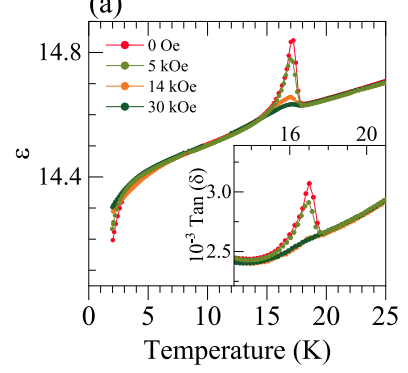

(b)

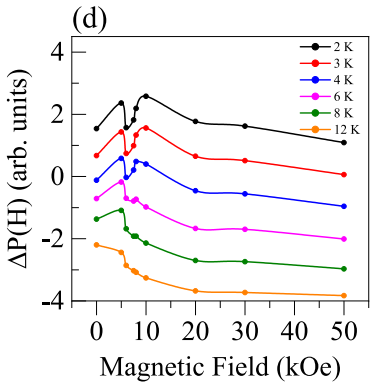

(c)

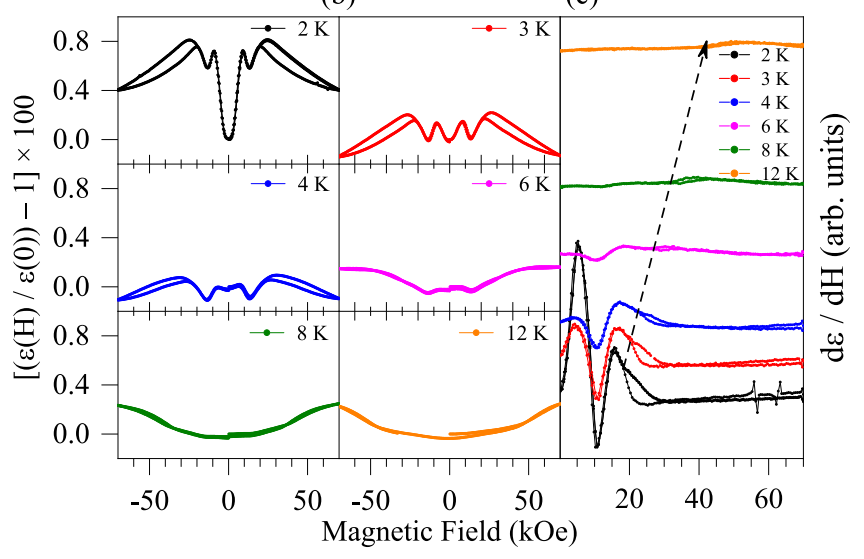

FIG. 5. (a) Temperature-dependent dielectric constant under different magnetic fields measured under the field-cooled condition, with the tangent loss, $\operatorname{Tan}(\delta)$, in the inset. The data were collected at the frequency of $f=1 \mathrm{kHz}$. (b) Field-dependent dielectric constant $\epsilon(H)$ at different temperatures, normalized by the expression $[\epsilon(H) / \epsilon(0)-1] \times 100,(c)$ Derivative of $\epsilon(H)$ at different temperatures with respect to the field. The curves are shifted vertically by distances equal to their temperature differences for clarity. The arrow shows the linear shift in the hysteresis toward higher fields as the temperature is increased. (d) Electric polarization as a function of field, $\Delta P(H)$, at different temperatures.

temperature advances to order as indicated by a peak seen in $\chi_{\mathrm{AC}}$ at $T=3.5 \mathrm{~K}$. It was observed for $\mathrm{DyFeWO}_{6}$ through neutron diffraction measurements that the ordering of $\mathrm{Dy}^{3+}$ spins is induced, through a cooperative process, by AFM ordering of $\mathrm{Fe}^{3+}$ at $T_{N}^{F e}$ [28]. The peak at $T=3.5 \mathrm{~K}$ can be suppressed with a magnetic field of $1 \mathrm{kOe}$. Increasing the magnetic field to $1 \mathrm{~T}$ causes an uptick in the susceptibility below $T_{N}$. Above $1 \mathrm{~T}$, a broad anomaly begins to appear from the lowest temperature measured and shifts to higher temperatures with increasing field. This could indicate a possible change in the magnetic structure. The $\chi_{\mathrm{AC}}(T)$ was also measured at two additional frequencies $(f=10 \mathrm{~Hz}$ and $100 \mathrm{~Hz}$ ) without external field, and the results are shown in Fig. S3 [42]. The data, which largely overlap within the resolution of our measurement, indicate a lack of glassy behavior.

Field-dependent DC magnetization, $M(H)$, was measured at various temperatures and the results are presented in Fig. 4(a). The data below $T_{\mathrm{N}}$ are in agreement with the AFM nature of the sample and the metamagnetic behavior at low temperature indicates the competing interaction between the two magnetic sublattices, $\mathrm{Ho}^{3+}$ and $\mathrm{Fe}^{3+}$, present in the sample. This is more evident from the derivative of magnetization, 
$d M / d H$, as shown in Fig. 4(b), where the two peaks at $2 \mathrm{~K}$ could be due to a spin-flop transition and seem to weaken and merge as the temperature is increased. As shown by the isothermal AC susceptibility results in Fig. S4 [42], this transition is rather continuous. The emergence of this spin-flop phase coincides with the ordering temperature of the $\mathrm{Ho}^{3+}$ spins, suggesting that $\mathrm{Ho}^{3+} 4 f$ spins and their interaction with $\mathrm{Fe}^{3+} 3 d$ spins play an important role in the observed metamagnetic behavior. The maximum moment that the sample gains at the lowest measured temperature and the highest applied field is $\sim 4.9 \mu_{\text {eff }} /$ f.u., which is lower than the theoretical value, indicating the presence of unsaturated magnetic moments.

Figures 3(e) and 5(a) summarize the effect of the magnetic field on the electric polarization and the dielectric constant, respectively. The magnetic field has a strong influence on electric polarization and suppresses the peak in the dielectric constant at $17.8 \mathrm{~K}$. The responses of both the dielectric constant and the polarization to the magnetic field evince the magnetoelectric (ME) coupling in the system. The polarization at $H=0$ Oe slightly decreases below a maximum at $T \sim 8.5 \mathrm{~K}$. This is in contrast to the behavior of $\mathrm{YFeWO}_{6}$ [28] with a nonmagnetic rare-earth cation, in which the polarization saturates below $T_{N}$. The decrease in polarization occurs in the temperature region below $T_{\max }$, which implies that the development of the $\mathrm{Ho}^{3+}$ magnetic sublattice and its interaction with $\mathrm{Fe}^{3+}$ likely causes the decrease in the polarization.

We used isothermal field-dependent measurements of the dielectric constant to further investigate the ME coupling in this system. The results are summarized in Fig. 5(b), which shows the magnetocapacitance (MC) effect with double-hysteresis behavior, in direct correspondence with the magnetization results shown in Figs. 4(a) and 4(b). Figure 5(c) displays the derivative of MC with respect to the magnetic field and shows a notable hysteretic region below $6 \mathrm{~K}$ that seems to persist at higher temperatures and moves toward larger field in a linear fashion as the temperature is increased.

Using the $\Delta P(T)$ graphs in Fig. 3(e), we constructed a $\Delta P(H)$ graph as displayed in Fig. 5(d) and, interestingly enough, it also shows a direct correspondence with the magnetization data in Fig. 4(b) and the MC data in Fig. 5(b). Although the MC effect could be mediated by factors other than ME coupling, both the observed change in polarization by magnetic field and the $\Delta P(H)$ curves support the proposition that the observed $\mathrm{MC}$ effect in this compound is due to ME coupling. $\Delta P(H)$ below $\sim 6 \mathrm{~K}$ is qualitatively echoed by the $\mathrm{MC}$ and magnetization data. This indicates the possible role of mutual interplay between $\mathrm{Ho}^{3+} 4 f$ and $\mathrm{Fe}^{3+} 3 d$ spins in the observed change in the polarization under magnetic field below $\sim 6 \mathrm{~K}$.

\section{CONCLUSION}

We have studied the multiferroic properties of, and magnetoelectric coupling in, the type-II multiferroic $\mathrm{HoFeWO}_{6}$. Through XRD measurements, we found the crystal structure to belong to the polar space group $P n a 2_{1}$ and no structural phase transition was observed down to $8 \mathrm{~K}$. DFT calculations also confirmed that this compound is stable in the Pna2 structure. XAS and XMCD measurements show an oxidation state of $3+$ for the Fe and Ho cations. Simulation of $\mathrm{XMCD}$ data suggests that the $\mathrm{Fe}^{3+}$ spins are arranged in a noncollinear fashion at angles of $90^{\circ}$ with respect to each other. The AFM and ferroelectric ordering at $T_{N}=17.8 \mathrm{~K}$ confirm the multiferroic nature of this compound. All of these observations favor the AFM ordering of $\mathrm{Fe}^{3+}$ as the origin of the ferroelectric transition, with inverse DM interaction playing an important role in breaking the inversion symmetry. Magnetic susceptibility below $T_{N}$ shows a broad maximum at $T_{\max } \sim 10.8 \mathrm{~K}$, which usually relates to a magnetic system with reduced dimensionality, and the ordering of $\mathrm{Ho}^{3+}$ spins at $\sim 3.5 \mathrm{~K}$. The decrease in polarization below $T_{\max }$ also seems to be related to the low-dimensional nature of the $\mathrm{Ho}^{3+}$ spins. The responses of both the dielectric constant and the polarization to the magnetic field reveal the presence of ME coupling. We used the MC effect to indirectly investigate the ME coupling and found that the field-dependent dielectric constant shows a direct correspondence with magnetization data. The polarization response to the magnetic field, especially $\Delta P(H)$ and its correspondence with the $M(H)$ curves, further supports the presence of ME coupling in this compound. The magnetization and $\mathrm{MC}$ data below $\sim 6 \mathrm{~K}$, in particular, are qualitatively echoed by $\Delta P(H)$, which implies the effect of mutual interaction between $\mathrm{Ho}^{3+} 4 f$ and $\mathrm{Fe}^{3+} 3 d$ spins on the ME coupling. Considering the noncollinear arrangement of spins in this compound, spin-orbit coupling likely plays a crucial role in the observed ME coupling, although contributions from other possible factors, e.g. magnetostriction, cannot be ignored. A substantiated and definite description of the mechanism for ME coupling requires further detailed single-crystal study.

\section{ACKNOWLEDGMENTS}

The work at University of Houston (UH) is supported by U. S. Air Force Office of Scientific Research Grants FA9550-15-1-0236 and FA9550-20-1-0068, the T. L. L. Temple Foundation, the John J. and Rebecca Moores Endowment, and the State of Texas through the Texas Center for Superconductivity at the University of Houston. The XRD patterns were collected at the National Synchrotron Radiation Research Center at Taiwan. The synchrotron XAS/XMCD experiments were performed at the BOREAS beamline of the ALBA Synchrotron Light Facility in collaboration with ALBA staff. Computational resources were provided by the Extreme Science and Engineering Discovery Environment (XSEDE) [55] supported by the National Science Foundation (ACI-1548562) and the National Energy Research Scientific Computing (NERSC) Center, a DOE Office of Science User Facility supported by the Office of Science, U. S. Department of Energy, under Contract No. DE-AC02-05CH11231. Additional support for this work was provided through resources of the UHPC cluster managed by UH and acquired through NSF Award 1531814. The authors acknowledge the use of the Maxwell/Opuntia/Sabine Cluster and the advanced support from the Research Computing Data Core at UH. The work at National Sun Yat-Sen University was partially supported by the Ministry of Science and Technology of Taiwan under Grant No. MOST 109-2112-M-110-019. 
[1] G. A. Smolenskia and I. E. Chupis, Ferroelectromagnets, Soviet Physics Uspekhi 25, 475 (1982).

[2] T. Kimura, T. Goto, H. Shintani, K. Ishizaka, T. Arima, and Y. Tokura, Magnetic control of ferroelectric polarization, Nature (London) 426, 55 (2003).

[3] H. Katsura, N. Nagaosa, and A. V. Balatsky, Spin Current And Magnetoelectric Effect In Noncollinear Magnets, Phys. Rev. Lett. 95, 057205 (2005).

[4] M. Mostovoy, Ferroelectricity In Spiral Magnets, Phys. Rev. Lett. 96, 067601 (2006).

[5] N. A. Spaldin and M. Fiebig, The renaissance of magnetoelectric multiferroics, Science 309, 391 (2005).

[6] D. Khomskii, Classifying multiferroics: Mechanisms and effects, Physics 2, 20 (2009).

[7] B. Lorenz, Y. Q. Wang, Y. Y. Sun, and C. W. Chu, Large magnetodielectric effects in orthorhombic $\mathrm{HoMnO}_{3}$ and $\mathrm{YMnO}_{3}$, Phys. Rev. B 70, 212412 (2004).

[8] B. Lorenz, A. P. Litvinchuk, M. M. Gospodinov, and C. W. Chu, Field-Induced Reentrant Novel Phase and a FerroelectricMagnetic Order Coupling in $\mathrm{HoMnO}_{3}$, Phys. Rev. Lett. 92, 087204 (2004).

[9] B. Lorenz, F. Yen, M. M. Gospodinov, and C. W. Chu, Fieldinduced phases in $\mathrm{HoMnO}_{3}$ at low temperatures, Phys. Rev. B 71, 014438 (2005).

[10] M. Fiebig, Revival of the magnetoelectric effect, J. Phys. D 38, R123 (2005).

[11] W. Eerenstein, N. D. Mathur, and J. F. Scott, Multiferroic and magnetoelectric materials, Nature (London) 442, 759 (2006).

[12] R. P. Chaudhury, B. Lorenz, Y. Q. Wang, Y. Y. Sun, and C. W. Chu, Magnetic field induced ferroelectricity in $\mathrm{Mn}_{0.9} \mathrm{Fe}_{0.1} \mathrm{WO}_{4}$, J. Appl. Phys. 103, 07E312 (2008).

[13] N. Poudel, K.-C. Liang, Y.-Q. Wang, Y. Y. Sun, B. Lorenz, F. Ye, J. A. Fernandez-Baca, and C. W. Chu, Magneticfield-induced spontaneous polarization reversal in multiferroic $\mathrm{Mn}_{0.85} \mathrm{Co}_{0.15} \mathrm{WO}_{4}$, Phys. Rev. B 89, 054414 (2014).

[14] B. Lorenz, Hexagonal manganites- $\left(\mathrm{RMnO}_{3}\right)$ : class (I) multiferroics with strong coupling of magnetism and ferroelectricity, ISRN Condens. Matter Phys. 2013, 1 (2013).

[15] B. B. Van Aken, T. T. Palstra, A. Filippetti, and N. A. Spaldin, The origin of ferroelectricity in magnetoelectric $\mathrm{YMnO}_{3}$, Nat. Mater. 3, 164 (2004).

[16] M. Lilienblum, T. Lottermoser, S. Manz, S. M. Selbach, A. Cano, and M. Fiebig, Ferroelectricity in the multiferroic hexagonal manganites, Nat. Phys. 11, 1070 (2015).

[17] N. A. Spaldin, Multiferroics beyond electric-field control of magnetism, Proc. R. Soc. London, Ser. A 476, 20190542 (2020).

[18] Z. J. Huang, Y. Cao, Y. Y. Sun, Y. Y. Xue, and C. W. Chu, Coupling between the ferroelectric and antiferromagnetic orders in $\mathrm{YMnO}_{3}$, Phys. Rev. B 56, 2623 (1997).

[19] Y. Wang, G. L. Pascut, B. Gao, T. A. Tyson, K. Haule, V. Kiryukhin, and S.-W. W. Cheong, Unveiling hidden ferrimagnetism and giant magnetoelectricity in polar magnet $\mathrm{Fe}_{2} \mathrm{Mo}_{3} \mathrm{O}_{8}$, Sci. Rep. 5, 12268 (2015).

[20] T. Kurumaji, S. Ishiwata, and Y. Tokura, Doping-Tunable Ferrimagnetic Phase with Large Linear Magnetoelectric Effect in a Polar Magnet $\mathrm{Fe}_{2} \mathrm{Mo}_{3} \mathrm{O}_{8}$, Phys. Rev. X 5, 031034 (2015).

[21] T. Kurumaji, S. Ishiwata, and Y. Tokura, Diagonal magnetoelectric susceptibility and effect of Fe doping in the polar ferrimagnet $\mathrm{Mn}_{2} \mathrm{Mo}_{3} \mathrm{O}_{8}$, Phys. Rev. B 95, 045142 (2017).
[22] V. Caignaert, A. Maignan, K. Singh, C. Simon, V. Pralong, B. Raveau, J. F. Mitchell, H. Zheng, A. Huq, and L. C. Chapon, Gigantic magnetic-field-induced polarization and magnetoelectric coupling in a ferrimagnetic oxide $\mathrm{CaBaCo}_{4} \mathrm{O}_{7}$, Phys. Rev. B 88, 174403 (2013).

[23] R. D. Johnson, K. Cao, F. Giustino, and P. G. Radaelli, $\mathrm{CaBaCo}_{4} \mathrm{O}_{7}$ : A ferrimagnetic pyroelectric, Phys. Rev. B 90, 045129 (2014).

[24] K. Singh, V. Caignaert, L. C. Chapon, V. Pralong, B. Raveau, and A. Maignan, Spin-assisted ferroelectricity in ferrimagnetic $\mathrm{CaBaCo}_{4} \mathrm{O}_{7}$, Phys. Rev. B 86, 024410 (2012).

[25] Y. S. Oh, S. Artyukhin, J. J. Yang, V. Zapf, J. W. Kim, D. Vanderbilt, and S. W. Cheong, Non-hysteretic colossal magnetoelectricity in a collinear antiferromagnet, Nat. Commun. 5, 3201 (2014).

[26] I. Živković, K. Prša, O. Zaharko, and $\mathrm{H}$. Berger, $\mathrm{Ni}_{3} \mathrm{TeO}_{6}-$ a collinear antiferromagnet with ferromagnetic honeycomb planes, J. Phys.: Condens. Matter 22, 056002 (2010).

[27] L. Zhao, C.-H. Du, and A. C. Komarek, Spin-driven pyroelectricity in $\mathrm{Ni}_{3} \mathrm{TeO}_{6}$ without ferroelectric signatures of the transition at Néel temperature, physica status solidi (RRL) Rapid Research Letters 11, 1700073 (2017).

[28] S. Ghara, E. Suard, F. Fauth, T. T. Tran, P. S. Halasyamani, A. Iyo, J. Rodríguez-Carvajal, and A. Sundaresan, Ordered aeschynite-type polar magnets $\mathrm{RFeWO}_{6}(\mathrm{R}=\mathrm{Dy}, \mathrm{Eu}, \mathrm{Tb}$, and Y): A new family of type-II multiferroics, Phys. Rev. B 95, 224416 (2017).

[29] P. N. Ravi Shankar, S. Mishra, and S. Athinarayanan, Polar magnetic oxides from chemical ordering: A new class of multiferroics, APL Mater. 8, 040906 (2020).

[30] S. W. Kim, T. J. Emge, Z. Deng, R. Uppuluri, L. Collins, S. H. Lapidus, C. U. Segre, M. Croft, C. Jin, V. Gopalan, S. V. Kalinin, and M. Greenblatt, $\mathrm{YCrWO}_{6}$ : polar and magnetic oxide with $\mathrm{CaTa}_{2} \mathrm{O}_{6}$-related structure, Chem. Mater. 30, 1045 (2018).

[31] R. Salmon, H. Baudry, J. Grannec, and G. L. Flem, Sur de nouvelles séries de composés du tungstène +IV de type aeschynite, Rev. Chim. Miner. 11, 71 (1974).

[32] B. H. Toby and R. B. Von Dreele, GSAS-II: The genesis of a modern open-source all purpose crystallography software package, J. Appl. Crystallogr. 46, 544 (2013).

[33] A. Barla, J. Nicolás, D. Cocco, S. M. Valvidares, J. HerreroMartín, P. Gargiani, J. Moldes, C. Ruget, E. Pellegrin, and S. Ferrer, Design and performance of BOREAS, the beamline for resonant $\mathrm{X}$-ray absorption and scattering experiments at the ALBA synchrotron light source, J. Synchrotron Radiat. 23, 1507 (2016).

[34] G. Kresse and J. Hafner, Ab initio molecular-dynamics simulation of the liquid-metal-amorphous-semiconductor transition in germanium, Phys. Rev. B 49, 14251 (1994).

[35] G. Kresse and J. Hafner, Ab initio molecular dynamics for liquid metals, Phys. Rev. B 47, 558 (1993).

[36] G. Kresse and J. Furthmüller, Efficiency of ab-initio total energy calculations for metals and semiconductors using a plane-wave basis set, Comput. Mater. Sci. 6, 15 (1996).

[37] A. H. Larsen, J. J. Mortensen, J. Blomqvist, I. E. Castelli, R. Christensen, M. Dułak, J. Friis, M. N. Groves, B. Hammer, and C. Hargus, The atomic simulation environment-a Python library for working with atoms, J. Phys.: Condens. Matter 29, 273002 (2017). 
[38] J. P. Perdew, K. Burke, and M. Ernzerhof, Generalized Gradient Approximation Made Simple, Phys. Rev. Lett. 77, 3865 (1996).

[39] P. E. Blöchl, Projector augmented-wave method, Phys. Rev. B 50, 17953 (1994).

[40] G. Kresse and D. Joubert, From ultrasoft pseudopotentials to the projector augmented-wave method, Phys. Rev. B 59, 1758 (1999).

[41] J. Heyd, G. E. Scuseria, and M. Ernzerhof, Hybrid functionals based on a screened Coulomb potential, J. Chem. Phys. 118, 8207 (2003).

[42] See Supplemental Material at http://link.aps.org/supplemental/ 10.1103/PhysRevB.103.094110 for the details of sum rule results, spin magnetic moments by DFT calculations, crystal structure, and magnetic data.

[43] C. T. Chen and F. Sette, High resolution soft x-ray spectroscopies with the dragon beamline, Phys. Scr. T31, 119 (1990).

[44] C. Mitra, Z. Hu, P. Raychaudhuri, S. Wirth, S. I. Csiszar, H. H. Hsieh, H.-J. Lin, C. T. Chen, and L. H. Tjeng, Direct observation of electron doping in $\mathrm{La}_{0.7} \mathrm{Ce}_{0.3} \mathrm{MnO}_{3}$ using x-ray absorption spectroscopy, Phys. Rev. B 67, 092404 (2003).

[45] T. Burnus, Z. Hu, M. W. Haverkort, J. C. Cezar, D. Flahaut, V. Hardy, A. Maignan, N. B. Brookes, A. Tanaka, H. H. Hsieh, H.-J. Lin, C. T. Chen, and L. H. Tjeng, Valence, spin, and orbital state of $\mathrm{Co}$ ions in one-dimensional $\mathrm{Ca}_{3} \mathrm{Co}_{2} \mathrm{O}_{6}$ : An x-ray absorption and magnetic circular dichroism study, Phys. Rev. B 74, 245111 (2006).

[46] T. Burnus, Z. Hu, H. H. Hsieh, V. L. J. Joly, P. A. Joy, M. W. Haverkort, H. Wu, A. Tanaka, H.-J. Lin, C. T. Chen, and L. H. Tjeng, Local electronic structure and magnetic properties of $\mathrm{LaMn}_{0.5} \mathrm{Co}_{0.5} \mathrm{O}_{3}$ studied by $\mathrm{x}$-ray absorption and magnetic circular dichroism spectroscopy, Phys. Rev. B 77, 125124 (2008).

[47] B. T. Thole, P. Carra, F. Sette, and G. van der Laan, X-Ray Circular Dichroism As A Probe Of Orbital Magnetization, Phys. Rev. Lett. 68, 1943 (1992).

[48] P. Carra, B. T. Thole, M. Altarelli, and X. Wang, X-Ray Circular Dichroism And Local Magnetic Fields, Phys. Rev. Lett. 70, 694 (1993).

[49] C. Piamonteze, P. Miedema, and F. M. F. de Groot, Accuracy of the spin sum rule in XMCD for the transition-metal L edges from manganese to copper, Phys. Rev. B 80, 184410 (2009).

[50] A. Tanaka and T. Jo, Resonant 3d, 3p and 3s photoemission in transition metal oxides predicted at $2 \mathrm{p}$ threshold, J. Phys. Soc. Jpn. 63, 2788 (1994).

[51] W. A. Harrison, Electronic Structure and the Properties of Solids: The Physics of the Chemical Bond, Dover Books on Physics (Dover Publications, New York, 1989).

[52] $\mathrm{FeO}_{6}$ cluster parameters $[e V]: \mathrm{U}_{d d}=6.0, \mathrm{U}_{p d}=7.5$, charge transfer energy $\Delta_{\mathrm{CT}}=2.0$, ionic crystal field $10 \mathrm{Dq}=0.7, \quad$ hybridization $\quad V\left(x^{2}-y^{2}\right)=V\left(z^{2}\right)=2.69$, $V(x y)=V(x z)=V(y z)=1.51, \quad$ ligand crystal field $10 \mathrm{Dq}_{\text {lig }}=1.0$, spin-orbit coupling $\zeta_{3 d}=0.059$, exchange field $H_{\text {ex }}=0.004$, and magnetic field $6 \mathrm{~T}$. The Slater integrals were reduced to $75 \%$ of Hartree-Fock values.

[53] Y. Teramura, A. Tanaka, B. T. Thole, and T. Jo, Effect of coulomb interaction on the X-ray magnetic circular dichroism spin sum rule in rare earths, J. Phys. Soc. Jpn. 65, 3056 (1996).

[54] A. N. Vasiliev, O. S. Volkova, E. A. Zvereva, and M. Markina, Low-Dimensional Magnetism (CRC Press, Boca Raton, 2019).

[55] J. Towns, T. Cockerill, M. Dahan, I. Foster, K. Gaither, A. Grimshaw, V. Hazlewood, S. Lathrop, D. Lifka, G. D. Peterson et al., Xsede: accelerating scientific discovery, Comput. Sci. Eng. 16, 62 (2014). 